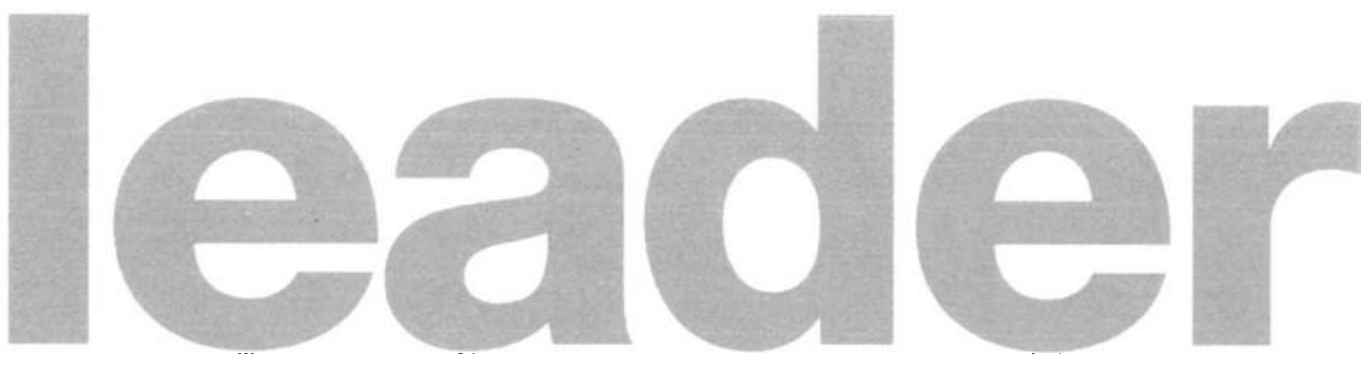

\title{
Beyond the Research Assessment Exercise
}

Following the 1996 Research Assessment Exercise (p6), schools of Architecture in the UK and elsewhere are facing the fact that their financial well-being and possibly even their existence depends on demonstrating that they are doing a considerable volume of high-grade research whether or not such an activity has characterised their culture in the past.

Central to the agonised discussion that has arisen (arq vol. 1 nos. 1 and 2) has been the question: Is design not research? Whether or not a positive answer to this question would be the salvation of schools - as is sometimes implied - is an open question, but the debate does provoke wider questions about the advancement of architectural knowledge and the roles of the various actors in its unfolding story. Such questions might be: How does architectural knowledge advance? Are buildings better or worse than they used to be: is progress being made? What might 'progress' mean in this context? To reflect on such questions it is necessary to recognise that within the production of buildings-and-theirarchitecture, there are diverse aspects in respect of which quite different answers to such questions might be given.

To discover whether, in this production, research done in universities has a leading role, a walk-on part or even a place in the prompt corner, it has to be seen in the context of the other players. For example, at centre stage is the innovative designer who, in a profession with few leaders and many who are led, may bring about far-reaching changes. Investigation may, however, reveal that such innovation has been crucially influenced by the scholarly writing of an academic theorist or historian or by similar ideas mediated by educators. In a parallel way, the undoubted effects on buildings of changed legislation may well be traced to university research in architectural sciences. Interactions between practice, research, scholarship and teaching therefore deserve more examination.

To pursue the theatrical analogy one step further - what about the paying public? Their confidence can only be retained - some would say regained - if the design of buildings can be seen to be as an autonomous 'learning system' that by its own momentum and practices is seen to improve its performance. Within such a scenario there has to be the 
evaluation of current success and failure. And yet it is in this area that the architectural community has most obviously failed to advance.

Our understanding of recently-completed buildings seems to be lacking in at least two centrally-important respects - which turn out to be those much-loved, if sometimes neglected, characters of the commedia dell'architettura, commodity and delight.

The problem of assessing buildings to evaluate functional success is apparently resistant (pace Space Syntax) to the development of methods of appraisal and reporting that can be applied with any sort of ease. Understanding building use involves considerable conceptual difficulties and the procedures could well be tedious, but the architectural world ought surely to be able to demonstrate its ability to create objects that are valuable in terms of utility.

Writing openly and at length - and critically - about subtle but nevertheless real experiences that may arise from being in and using particular new buildings is not done as much as it might be in either the professional or the lay press. I hope that arq will continue to provide a forum for the objective and critically-supportive appraisal of buildings. It already brings practitioners, researchers and teachers together in a common intellectual arena. On this basis it can lead a collaborative quest for the elaboration of architectural building design as an autonomous learning activity.

From such a discourse, schools of Architecture may come to appreciate more fully their actual and potential contribution to the advancement of architectural knowledge and practice through their activities of education, research and scholarship, not only in response to audits by their paymasters but also as a process of self-development within a more reflective architectural community. 